\title{
Growth differentiation factor 15 (GDF15) is associated with non-alcoholic fatty liver disease (NAFLD) in youth with overweight or obesity
}

\author{
Brittany Galuppo ${ }^{1,3}$, Cristiana Agazzi $\mathbb{D}^{1,3}$, Bridget Pierpont ${ }^{1}$, Jennifer Chick ${ }^{1}$, Zhongyao Li ${ }^{1}$, Sonia Caprio ${ }^{1}$ and Nicola Santoro $\mathbb{D}^{1,2}{ }^{凶}$
}

(c) The Author(s) 2022

OBJECTIVE: Growth differentiation factor 15 (GDF15) has been associated with food intake and weight regulation in response to metabolic stress. In animal models, it has been noted that it may play a role in the progression of non-alcoholic fatty liver disease (NAFLD), the leading cause of chronic liver disease in children.

DESIGN: In the current study, we explored the association of circulating plasma concentrations of GDF15 with NAFLD in youth with overweight/obesity, and whether changes in plasma concentrations in GDF15 parallel the changes in intrahepatic fat content (HFF\%) over time.

METHODS: Plasma GDF15 concentrations were measured by ELISA in 175 youth with overweight/obesity who underwent an oral glucose tolerance test (OGTT) and magnetic resonance imaging (MRI) to assess intrahepatic, visceral, and subcutaneous fat. Baseline fasting GDF15 concentrations were measured in twenty-two overweight/obese youth who progressed $(n=11)$ or regressed $(n=11)$ in HFF\% by more than $30 \%$ of original over a 2 -year period.

RESULTS: Youth with NAFLD had significantly higher plasma concentrations of GDF15 than those without NAFLD, independent of age, sex, ethnicity, BMI $z$-score $(\mathrm{BMI})$, and visceral fat $(P=0.002)$. During the OGTT, there was a decline in plasma GDF15 concentrations from 0 to $60 \mathrm{~min}$, but GDF15 concentrations returned to basal levels by the end of the study. There was a statistically significant association between change in HFF\% and change in GDF15 $\left(P=0.008 ; r^{2}=0.288\right)$ over $\sim 2$ years of follow-up.

CONCLUSIONS: These data suggest that plasma GDF15 concentrations change with change in intrahepatic fat content in youth with overweight/obesity and may serve as a biomarker for NAFLD in children.

Nutrition and Diabetes (2022)12:9; https://doi.org/10.1038/s41387-022-00187-2

\section{INTRODUCTION}

Non-alcoholic fatty liver disease (NAFLD) is the most common liver disease in pediatrics and affects $\sim 30 \%$ of children and adolescents with obesity in the United States [1-4]. Despite the pervasiveness and severity of the disease, little is known about its pathogenesis and biomarkers associated with its onset and development. Recently, metabolic research has focused on growth differentiation factor 15 (GDF15), a stress-induced cytokine belonging to the transforming growth factor beta superfamily [5]. As of today, GDF15 has been associated with many conditions including cardiac dysfunction, metformin response, and tumor-related cachexia [6-8]. Metabolic effects of GDF15 are mediated by GDNF family receptor a-like (GFRAL), an endogenous receptor, uniquely expressed at the level of the central nervous tissue [9]. GDF15, however, is up-regulated in almost any cell or tissue in response to stress, thus suggesting its key role as an anti-inflammatory cytokine $[10,11]$.

Recent studies have shown that GDF15 is associated with NAFLD in adults. In particular, GDF15 represents an independent determinant of fibrosis severity in NAFLD: (1) patients with more severe chronic liver diseases have proportionately higher GDF15 values and (2) plasma GDF15 concentrations are associated with liver fibrosis regardless of diabetes status $[12,13]$.

So far, there are no studies investigating whether plasma GDF15 concentrations in youth with obesity are associated with NAFLD. In this study, we sought to determine whether GDF15 plasma concentrations are associated with (1) intrahepatic fat content, (2) changes in intrahepatic fat accumulation over time, and (3) the metabolic phenotype related to NAFLD in youth with obesity. To achieve our aims, we measured GDF15 in 175 youth with overweight/obesity who underwent an oral glucose tolerance test and an MRI to measure intrahepatic fat content. Moreover, we analyzed changes in GDF15 over time in 22 youth who were followed-up for $\sim 2$ years and also showed changes (increase or decrease) in intrahepatic fat content of at least $30 \%$. Herein, we show that in youth with overweight/obesity, GDF15 plasma concentration is associated with NAFLD and NAFLD-related phenotypes and that changes in plasma GDF15 concentration are driven by changes in intrahepatic fat content.

\footnotetext{
'Department of Pediatrics, Yale School of Medicine, New Haven, CT, USA. ²Department of Medicine and Health Sciences, "V. Tiberio," University of Molise, Campobasso, Italy.
} ${ }^{3}$ These authors contributed equally: Brittany Galuppo, Cristiana Agazzi. ${ }^{凶}$ email: nicola.santoro@yale.edu 
Table 1. The clinical characteristics of the cross-sectional cohort grouped by presence or absence of NAFLD are shown.

\begin{tabular}{|c|c|c|c|}
\hline & Youth without NAFLD $(n=89)$ & Youth with NAFLD $(n=86)$ & $P$-value \\
\hline Age (y) & $13.1 \pm 3.09$ & $13.6 \pm 3.03$ & 0.224 \\
\hline $\operatorname{Sex}(M / F)$ & $(27 / 62)$ & $(42 / 44)$ & 0.174 \\
\hline Ethnicity $(\mathrm{C} / \mathrm{AA} / \mathrm{H} / \mathrm{O})$ & $21 / 35 / 29 / 4$ & $17 / 8 / 54 / 7$ & $<1.0 \times 10^{-10}$ \\
\hline BMIz & $2.23 \pm 0.461$ & $2.37 \pm 0.367$ & 0.145 \\
\hline Fasting glucose (mg/dL) & $90 \pm 7$ & $92 \pm 8$ & 0.039 \\
\hline Fasting insulin (mU/L) & $31 \pm 24$ & $41 \pm 26$ & $2.32 \times 10^{-4}$ \\
\hline WBISI & $2.25 \pm 1.14$ & $1.56 \pm 0.895$ & $3.65 \times 10^{-5}$ \\
\hline Triglycerides (mg/dL) & $103 \pm 43.8$ & $170 \pm 145$ & $3.75 \times 10^{-4}$ \\
\hline $\mathrm{HDL}(\mathrm{mg} / \mathrm{dL})$ & $43.1 \pm 8.48$ & $40.5 \pm 10.5$ & 0.044 \\
\hline LDL (mg/dL) & $93.8 \pm 27.5$ & $97.9 \pm 24.5$ & 0.361 \\
\hline Total cholesterol (mg/dL) & $161 \pm 30.5$ & $169 \pm 40.7$ & 0.316 \\
\hline VAT/VAT + SAT & $0.114 \pm 0.038$ & $0.153 \pm 0.047$ & $2.22 \times 10^{-7}$ \\
\hline
\end{tabular}

A Mann-Whitney test was used to compare the differences between subjects with and without NAFLD. Glucose, insulin, triglycerides, HDL, LDL, total cholesterol, and ALT were measured from plasma. Data are shown as mean \pm standard deviation.

$A A$ African American, $C$ Caucasian, $F$ female, $H$ Hispanic, $M$ male, $O$ Other, WBISI whole body insulin sensitivity index. Italics values identify statistical significance.

\section{SUBJECTS AND METHODS Study population}

A cross-sectional cohort of children and adolescents with overweight/obesity was identified from the Yale Pediatric Obesity Clinic main research study cohort (New Haven, CT). A multiethnic group of 175 youth with overweight/ obesity between the ages of 8 and 21 years was selected based on protocol inclusion and exclusion criteria. Per the study protocol, participants had a $\mathrm{BMI} \geq 85^{\text {th }}$ percentile according to U.S. Centers for Disease Control and Prevention (CDC) growth charts [14], and were excluded for the use of medication on a chronic basis, presence of a chronic condition, baseline blood creatinine $>1.0 \mathrm{mg} / \mathrm{dL}$, pregnancy, presence of endocrinopathies, or use of metformin. Subjects were otherwise healthy children and adolescents.

Baseline fasting plasma GDF15 concentrations were measured in all subjects and in a subgroup of subjects with $(n=10)$ and without $(n=10)$ NAFLD, matched for age, sex, BMIz, and ethnicity, during an OGTT. Longitudinal analyses were performed in a subgroup of 22 youth with overweight/obesity who were followed up for $\sim 2$ years ( 1.79 years \pm 0.178 ) and either increased $(n=11)$ or decreased $(n=11)$ HFF\% by more than $30 \%$ in the two-year period. All subjects underwent an oral glucose tolerance test and abdominal MRI to measure intrahepatic fat content (HFF $\%)$, visceral, and subcutaneous fat as previously described $[2,15]$. The study was approved by the Yale University Human Investigations Committee in accordance with the Helsinki Declaration of 1975 as revised in 1983 . Written consent was obtained from each patient after full explanation of the purpose and nature of all procedures used.

\section{Biochemical analyses}

Plasma GDF15 concentrations were measured using Human Quantikine ELISA kits (SGD150, R\&D Systems Inc., Minneapolis, MN, USA) according to manufacturer's instructions. The detection threshold for this kit is $4.39 \mathrm{pg} /$ $\mathrm{mL}$ and the assay range is $23.4-1,500 \mathrm{pg} / \mathrm{mL}$. Plasma glucose was measured at bedside during the OGTT using the YSI2700-STAT-Analyzer (Yellow Springs Instruments, Yellow Springs, OH, USA), plasma insulin was measured by antibody radioimmunoassay from Millipore Sigma (Billerica, MA, USA), and lipid levels were measured using Auto-Analyzer (model 747200, Roche Diagnostics, Indianapolis, IN, USA).

\section{Statistical analyses}

Categorical data are presented as counts (percent) and were analyzed using a $x^{2}$ test. Continuous variables are presented as mean (standard deviation) or as median (interquartile range) for HFF\%. Non-normally distributed variables were log-transformed to approximate normality, except for HFF\% that was transformed into rank (PROC RANK). A MannWhitney test was used to compare the differences between subjects with and without NAFLD and to compare differences between the crosssectional cohort and the subgroup for longitudinal follow-up. A general linear model (PROC GLM) was used to test differences between NAFLD groups, and age, sex, ethnicity, BMIz, and visceral adipose tissue (VAT) were used as covariates. A GLM was also used to assess the association of GDF15 with VAT, using age, sex, ethnicity, BMIz, and HFF\% as covariates. A simple regression analysis was used to test the association between change in intrahepatic fat content and change in GDF15 concentration. Statistical significance was established at an alpha of 0.05 . Analyses were performed using SAS 9.4 (Cary, NC) and GraphPad Prism software 9.0.0 (San Diego, CA).

\section{RESULTS}

\section{GDF15 in Youth with and without NAFLD}

In Table 1, the clinical characteristics of study participants stratified by presence or absence of NAFLD, as defined by HFF\% greater than or equal to $5.5 \%$, are shown. Of the 175 youth with overweight/obesity included in the study, 89 youth did not have NAFLD and 86 youth demonstrated the MRI features of NAFLD. There were no statistically significant differences between the two groups in terms of age $(P=0.224)$ and sex $(P=0.174)$. BMI $z$-score, measures of glucose metabolism and insulin sensitivity, and ALT levels were higher in the group with NAFLD. Fasting plasma GDF15 levels were significantly different between the two groups, independent from age, sex, BMI $z$-score, ethnicity, and VAT $(P=$ 0.002 ) (Fig. 1A). No association was found between GDF15 levels and BMlz $(P=0.085)$ (Supplemental Fig. 1).

GDF15 was associated with VAT independent of age, sex, ethnicity, and BMIz $(P=0.008)$, but after adjustment for intrahepatic fat content, the association became marginally significant $(P=0.042)$.

Similarly, there was also an association between WBISI and GDF15 independent from age, sex, ethnicity, and BMIz $(p=0.006)$, which disappeared after adjusting for HFF\% $(P=0.082)$. 
A

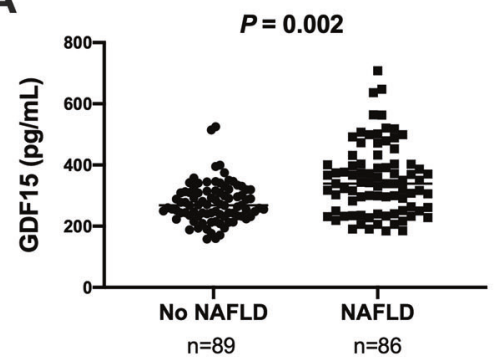

B

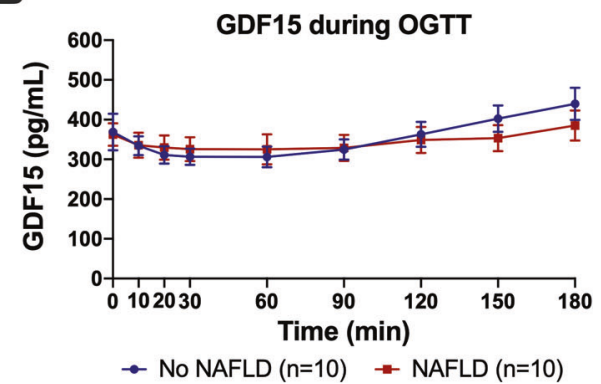

Fig. 1 GDF15 in a cross-sectional cohort of youth with and without NAFLD. A Fasting plasma GDF15 concentration in overweight/obese youth with or without NAFLD. P-value is from Mann-Whitney test. B Plasma GDF15 levels during an OGTT in overweight/obese youth with and without NAFLD, matched for age, sex, ethnicity, BMIz. Youth without NAFLD are depicted in blue and youth with NAFLD are depicted in red. Differences in GDF15 concentrations during an OGTT between the groups were not statistically significant. Error bars represent standard error of the mean.

A

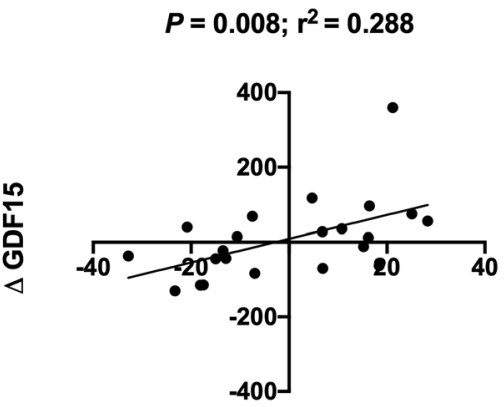

$\Delta$ HFF\%
B

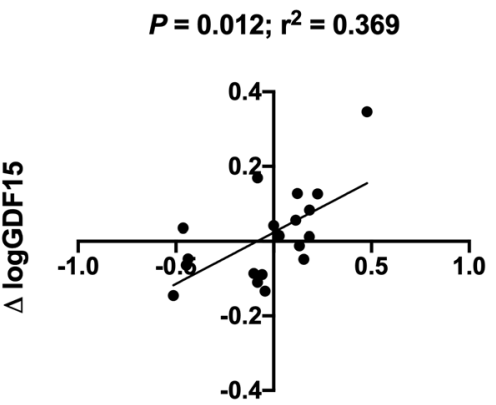

$\triangle \log A L T$

Fig. 2 Change in GDF15 in youth that increased or decreased in HFF\% by at least $30 \%$ of original in a two-year period. A Correlation between change in HFF\% and change in plasma GDF15 concentrations. B Correlation between change in logALT concentrations and change in logGDF15 concentrations. $P$-values and $r^{2}$ values are from Spearman rank correlation.

We measured plasma GDF15 during a 3-h OGTT and observed a slight reduction in GDF15 concentrations from 0 to $60 \mathrm{~min}$. The GDF15 concentrations tended to have a greater initial reduction $(0-60 \mathrm{~min})$ and later rebound $(60-180 \mathrm{~min})$ in the group without NAFLD compared to the group with NAFLD (Fig. 1B). The delta change in GDF15 concentration from baseline $(0 \mathrm{~min})$ to the nadir (60 min) was similar between the groups with $(81.2 \pm 39.0)$ and without $(58.5 \pm 17.7)$ NAFLD $(P=0.971)$.

\section{Changes in GDF15 over time}

A subgroup of 22 youth with obesity who increased or decreased in HFF\% by at least $30 \%$ of original over a two-year period was assessed for changes in plasma GDF15 concentrations. The subgroup did not differ significantly from the cross-sectional cohort in terms of age, sex, and BMIz, but differed significantly in measures of glucose and insulin metabolism, as most subjects in the subgroup had NAFLD to begin with. In the subgroup, there was an association between delta change in HFF\% and delta change in plasma GDF15 concentrations, in which the change in GDF15 paralleled the change in HFF\% $\left(P=0.008 ; r^{2}=0.288\right)$ (Fig. 2A). Similar observations were made for change in plasma GDF15 concentrations and changes in plasma ALT concentrations $\left(P=0.012 ; r^{2}=0.369\right)$ (Fig. 2B).

\section{DISCUSSION}

In the present study, we observed an association between plasma concentrations of GDF15 and NAFLD and an association between changes in HFF\% and changes in plasma GDF15 concentrations. Specifically, in subjects who experienced a reduction of NAFLD severity, plasma concentrations of GDF15 decreased, while, in those in whom the disease progressed, plasma concentrations of GDF15 increased significantly. Consistent with what has been shown before in adults, GDF15 concentrations in plasma tend to decrease during an OGTT and return to the initial concentrations by the end of the 3-hour study [16]. Other studies in adults have shown that GDF15 concentration tends to increase as the NAFLD phenotype worsens. Koo et al. [12] observed in a group of 190 adults with NAFLD, that GDF15 concentrations were higher in subjects with a greater degree of fibrosis. In our study, the observation that the degree of change in GDF15 paralleled the degree of change in intrahepatic fat content over time corroborates the hypothesis that GDF15 synthesis might increase with an increase of lipid synthesis in the liver. In a study that aimed to explore the role of GDF15 in the modulation of immune response, Luan et al. [17] showed that GDF15 concentrations are increased with increased production of lipids in the liver of a mouse model. In the study, the authors demonstrated that GDF15 may provide a protective effect against the inflammatory insult induced by viruses and bacteria and concluded that GDF15 attenuates the inflammatory response by modulating lipid metabolism [17].

It is likely that the upregulation of GDF15 might be a protective mechanism against the inflammatory response induced by free fatty acids in the context of fatty liver disease and the inflammation sustained by macrophages that leads to NASH and fibrosis. Moreover, Luan et al. [17] also showed that GDF15 infusion enhances hepatic triglycerides export into the circulation. If this is confirmed in humans, it may be another mechanism through which GDF15 may prevent liver damage in individuals 
with NAFLD. The hypotheses were generated by the findings from Luan et al. [17] seem to support the findings by Kim et al. [18], showing that GDF15 downregulates the expression of genes involved in the development of liver fibrosis and that the overexpression of GDF15 in transgenic mice alleviates hepatic inflammation and the NASH phenotype [18]. Furthermore, additional studies demonstrated that GDF15 expression in the liver can significantly promote beta-oxidation of fatty acids and ketogenesis in hepatocytes, thus playing a protective role against hepatic steatosis and inflammation [19,20].

We acknowledge that our study has some limitations. The main limitation of the study is that, although the association observed between GDF15 and hepatic fat is strong, this study does not include information about liver histology that would be needed to understand whether GDF15 is related to changes in liver histology such as inflammation and fibrosis. Moreover, we are not able to explain whether this association underlies a causal relationship, or that it is merely an epiphenomenon within the context of more complex metabolic changes. Therefore, larger longitudinal and interventional studies are warranted to shed light on the metabolic dynamics linking GDF15 and NAFLD in youth with overweight or obesity.

\section{REFERENCES}

1. Anderson EL, Howe LD, Jones HE, Higgins JP, Lawlor DA, Fraser A. The prevalence of non-alcoholic fatty liver disease in children and adolescents: a systematic review and meta-analysis. PLoS ONE. 2015;10:e0140908.

2. Trico D, Caprio S, Rosaria Umano G, Pierpont B, Nouws J, Galderisi A, et al. Metabolic features of nonalcoholic fatty liver (NAFL) in obese adolescents: findings from a multiethnic cohort. Hepatology. 2018;68:1376-90.

3. Marzuillo P, Del Giudice EM, Santoro N. Pediatric non-alcoholic fatty liver disease: new insights and future directions. World J Hepatol. 2014;6:217-25.

4. D'Adamo E, Santoro N, Caprio S. Metabolic syndrome in pediatrics: old concepts revised, new concepts discussed. Pediatr Clin North Am. 2011;58:1241-55. xi.

5. Yokoyama-Kobayashi M, Saeki M, Sekine S, Kato S. Human CDNA encoding a novel TGF-beta superfamily protein highly expressed in placenta. J Biochem. 1997;122:622-6.

6. Hsiao EC, Koniaris LG, Zimmers-Koniaris T, Sebald SM, Huynh TV, Lee SJ. Characterization of growth-differentiation factor 15, a transforming growth factor beta superfamily member induced following liver injury. Mol Cell Biol. 2000;20:3742-51.

7. Johnen $H$, Lin S, Kuffner T, Brown DA, Tsai VW, Bauskin AR, et al. Tumor-induced anorexia and weight loss are mediated by the TGF-beta superfamily cytokine MIC-1. Nat Med. 2007;13:1333-40.

8. Coll AP, Chen M, Taskar P, Rimmington D, Patel S, Tadross JA, et al. GDF15 mediates the effects of metformin on body weight and energy balance. Nature. 2020;578:444-8.

9. Emmerson PJ, Wang F, Du Y, Liu Q, Pickard RT, Gonciarz MD, et al. The metabolic effects of GDF15 are mediated by the orphan receptor GFRAL. Nat Med. 2017;23:1215-9.

10. Wallentin L, Hijazi Z, Andersson U, Alexander JH, De Caterina R, Hanna M, et al. Growth differentiation factor 15, a marker of oxidative stress and inflammation, for risk assessment in patients with atrial fibrillation: insights from the Apixaban for Reduction in Stroke and Other Thromboembolic Events in Atrial Fibrillation (ARISTOTLE) trial. Circulation. 2014;130:1847-58.

11. Lockhart SM, Saudek V, O'Rahilly S. GDF15: a hormone conveying somatic distress to the brain. Endocr Rev. 2020;41:bnaa007.

12. Koo BK, Um SH, Seo DS, Joo SK, Bae JM, Park JH, et al. Growth differentiation factor 15 predicts advanced fibrosis in biopsy-proven non-alcoholic fatty liver disease. Liver Int. 2018;38:695-705.

13. Lee ES, Kim SH, Kim HJ, Kim KH, Lee BS, Ku BJ. Growth differentiation factor 15 predicts chronic liver disease severity. Gut Liver. 2017;11:276-82.
14. Kuczmarski RJ, Ogden CL, Guo SS, Grummer-Strawn LM, Flegal KM, Mei Z, et al. 2000 CDC Growth Charts for the United States: methods and development. Vital Health Stat. 2002;11:1-190.

15. Umano GR, Caprio S, Di Sessa A, Chalasani N, Dykas DJ, Pierpont B, et al. The rs626283 variant in the MBOAT7 gene is associated with insulin resistance and fatty liver in caucasian obese youth. Am J Gastroenterol. 2018;113:376-83.

16. O'Rahilly S. GDF15-from biomarker to allostatic hormone. Cell Metab. 2017;26:807-8.

17. Luan HH, Wang A, Hilliard BK, Carvalho F, Rosen CE, Ahasic AM. et al. GDF15 Is an inflammation-induced central mediator of tissue tolerance. Cell. 2019;178:1231-44. e1211.

18. Kim KH, Kim SH, Han DH, Jo YS, Lee YH, Lee MS. Growth differentiation factor 15 ameliorates nonalcoholic steatohepatitis and related metabolic disorders in mice. Sci Rep. 2018;8:6789.

19. Zhang M, Sun W, Qian J, Tang Y. Fasting exacerbates hepatic growth differentiation factor 15 to promote fatty acid beta-oxidation and ketogenesis via activating XBP1 signaling in liver. Redox Biol. 2018;16:87-96.

20. Zhang $Z$, Xu X, Tian W, Jiang R, Lu Y, Sun Q, et al. ARRB1 inhibits non-alcoholic steatohepatitis progression by promoting GDF15 maturation. J Hepatol. 2020;72:976-89.

\section{ACKNOWLEDGEMENTS}

This work was supported by the National Institute of Health (R01-DK114504, R01MD015974 and R01-DK111038).

\section{AUTHOR CONTRIBUTIONS}

BG and CA collected and analyzed data, created figures, and wrote the manuscript. BG, BP, and JC recruited study participants. BP and JC collected data. SC and NS conceptualized the study, provided funding, and critically revised the manuscript.

\section{CONFLICT OF INTEREST}

The authors declare no competing interests.

\section{ADDITIONAL INFORMATION}

Supplementary information The online version contains supplementary material available at https://doi.org/10.1038/s41387-022-00187-2.

Correspondence and requests for materials should be addressed to Nicola Santoro.

Reprints and permission information is available at http://www.nature.com/ reprints

Publisher's note Springer Nature remains neutral with regard to jurisdictional claims in published maps and institutional affiliations.

(i) Open Access This article is licensed under a Creative Commons adaptation, distribution and reproduction in any medium or format, as long as you give appropriate credit to the original author(s) and the source, provide a link to the Creative Commons license, and indicate if changes were made. The images or other third party material in this article are included in the article's Creative Commons license, unless indicated otherwise in a credit line to the material. If material is not included in the article's Creative Commons license and your intended use is not permitted by statutory regulation or exceeds the permitted use, you will need to obtain permission directly from the copyright holder. To view a copy of this license, visit http://creativecommons. org/licenses/by/4.0/.

(c) The Author(s) 2022 